\title{
Image Preprocessing Methods Used In Meteorological Measurement of the
}

\section{Temperature Testing System}

\author{
Zhang Jiajia,Liang Ruyi.liuyu,Jiang Tao,Meng Xianluo,Wu Chunai \\ Meteorological technology and equipment center in Hebei Province \\ E-mail:sunyi_12_12@sina.com
}

\begin{abstract}
The Meteorological measurement automatic temperature testing system having a defect image. For example: noise problem, contrast is insufficient problem, put forward research programme for image pretreatment, try the median filter, histogram equalization, image binarization methods to remove noise, enhanced images for feature points clear and accurate. I have conducted simulation experiment for subsequent recognition process.
\end{abstract}

\section{Keywords - Thermometer image; Image pretreatment;}

Median filter; Histogram equalization ; Image binarization

$$
\text { I. Introduction }
$$

In order to get an accurate temperature effectively, we must first eliminate the effects of human factors, this is particularly important in the verification process of the glass thermometer. If you can see these human verification process to transfer images to a computer to extract the "mercury" This feature information, and by extracting feature information with a preset scale range for comparison, so as to achieve the purpose of extracting the exact scale value.

\section{Image preprocessing}

Temperature automated verification system is the most important part of image processing, and image pre-processing thermometer is the first step for image processing, Due to the different input image thermometer filming conditions, defects tend to noise, insufficient contrast and the like. In addition, the crew distance, focal length ranging in size also makes the level of information in the whole image position and size are not identified. Therefore desirable by the temperature obtained by the image data pre-processing to eliminate the influence of illumination, focal length, and distance to a certain extent on the conditions.

\section{Image denoising smoothing}

\section{A. Noise Types}

The actual images obtained are generally subject to some interference due to noisy. According to the relationship between noise and signal can be divided into additive noise and multiplicative noise, noisy image set said $f(x, y), g(x, y)$ which means that the original noise-free image, $n(x, y)$ which means that noise.

\section{1) Additive noise}

Such noise is independent of the input image signal, noisy image can be expressed as:

$$
f(x, \#) \quad g(x, y) \quad n(x, y)
$$

Noise generated by channel noise and scanned images are of this kind of noise in

2) Multiplicative noise

Such noise is related to the input image signal, noisy image can be expressed as: $f(x, y)=g(x, y)+n(x, y) g(x, y)$

\section{B. Image Denoising}

Commonly used in traditional spatial domain denoising main neighborhood average, median filtering, multiple images average method.

1) Neighborhood average

Neighborhood averaging method using the 
mean filter is applied to remove the image obtained by scanning the grain noise. In general, if the noise belongs to the additive noise, and is independent of white Gaussian noise (with zero mean and variance $\sigma^{2}$ ), SNR is defined as the ratio of noisy image of the mean and variance, Noisy image processing by neighborhood average, its signal to noise ratio will improve times (where $\mathrm{n}$ is the number of pixels contained in the neighborhood).

Visible taken neighborhood is, the more pixels, the more improved signal to noise ratio, the better the smoothing effect; From the perspective of the signal spectrum, the slowly varying part of the signal belongs to the low frequency portion in the frequency domain, the rapidly changing portion of the signal is high frequency portion in the frequency domain. If the average value of the neighborhood of the point of difference is not the case, then the point is not large noise, no smoothing to maintain boundary reduce blur. Gray solitary point for the noise and its neighborhood average gray disparity can be filtered out by this method.

\section{2) Median filter}

Median filter (Media filtering) is based on a sort of statistical theory can effectively suppress noise nonlinear signal processing technology. The advantage of this filter is simple and fast operation, showing the filtered white noise superimposed superimposed noise and long tail terms of better performance. Details (such as edges, acute, etc.) median filtering out noise while signals can be well protected. Further, an adaptive median filter easily, thereby further improving the filtering performance. Therefore, it is suitable for a number of linear filters not capable of digital image processing ${ }^{[1]}$.

The basic idea of the median filter is: For an image, each pixel in the image as the center point produces a rectangular sliding window (window size and generally odd),all the pixels within the window at the gray value from small to large order to sort and calculate the value of the sorted sequence to the center of the window instead of pixel values.Figure 1 is a comparison of the value after the image before and after filtering.
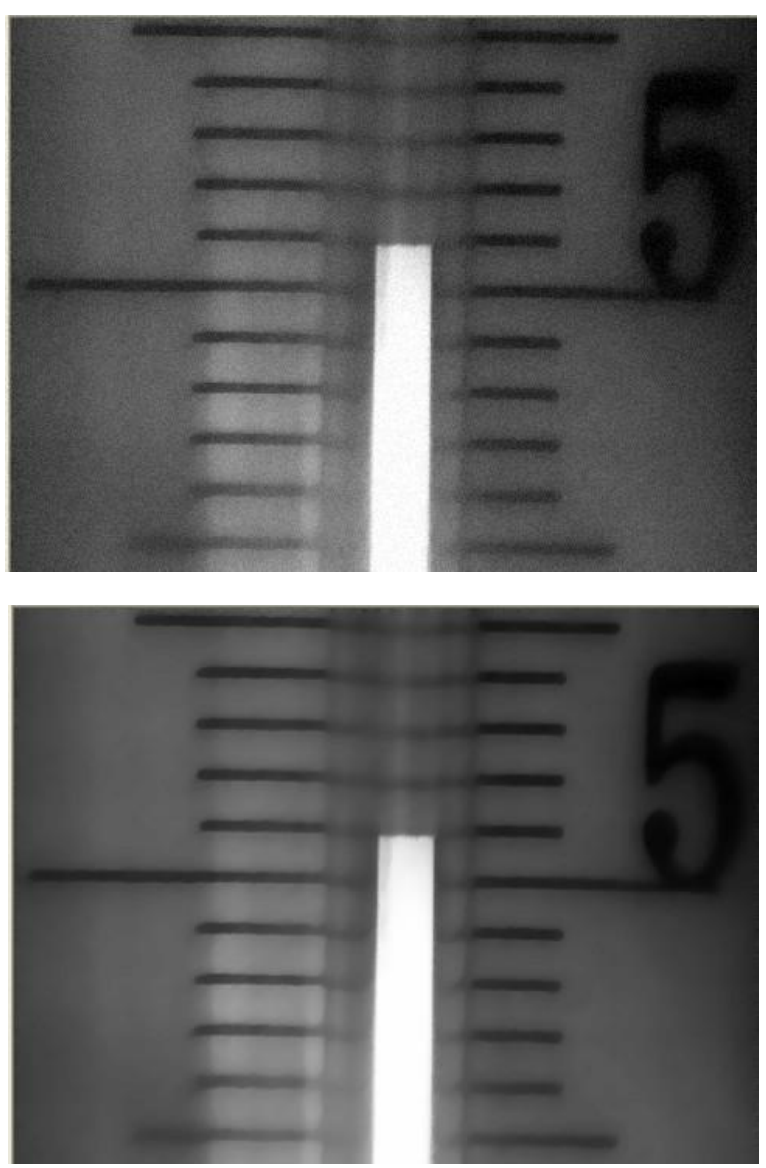

Figure 1. Comparison of median filtering the image after

Median overall median filter, it can effectively remove the image of a random shot noise, better to retain the original image transition section, median filtering denoising effect depends on the size of the filter window and participation the number of pixels calculated.

Filtering window has on the quality of the output image is greatly affected, the window is too small to de-noising effect is not good, the window will lose too much detail, resulting in blurred images. Some fine line images, and other details of the corner of the image that contains significant structural information, damage or loss of these structures is often more than the noise itself unacceptable. 


\section{3) Average multiple images}

Averaging a plurality of images of the same scene using multiple images to remove noise average, this method is the use of a plurality of images of the same scene and superimposed noise elimination, Video camera used in a television camera in order to reduce noise pickup tube or CCD photoelectric device caused, then the multiple images of the same scene taken continuously and digitized, and then the average of multiple images, 8 is generally used in the image averaging, in the practical application of this method is the difficulty of how the registration of multiple images together, so that the corresponding pixel corresponds correctly aligned.

\section{Image Enhancement}

Image enhancement technique is an extension of the image contrast, image processing visual optimization techniques. The main purpose of image enhancement is to improve the dynamic effects grayscale images, highlighting some of the information in the image, so that the processed image is more suitable for a particular application than the original image ${ }^{[2]}$.

There are two types of image enhancement methods: spatial domain method and frequency domain method.

The image enhancement method is mainly in the spatial domain to the spatial domain image pixel gray value direct calculation processing, the gray value of each point of a point contained in a small area for the average calculation, instead of using the average value of the obtained point gray value.

Image Enhancement Technology airspace described by the following equation:

$f(x, y)=g(x, y) \bullet h(x, y)$

$f(x, y) 、 g(x, y)$ Pre-processing the image, respectively, $h(x, y)$ after the space budget function.
The image enhancement method in a frequency domain transform domain image (typically in the frequency domain) of the image to a certain conversion value calculation, and then transformed back to the spatial domain, can be the Fourier transform of the image, and then the spectrum of the image to a correction (such as filtering), then the final image will be corrected inverse Fourier transformed back to the spatial domain, thereby enhancing the image.

A histogram is a function of gray level, which represents the image having the number of pixels of each gray level, the frequency of each reflected image appears gray. The abscissa is the histogram gray level, the frequency of the vertical axis is the gray-scale reflecting the basic statistical characteristics of the image.

Histogram equalization (gray balance) is an enhanced gray scale method, the basic idea of this method is the histogram of the original image is converted into the form of a uniform distribution, thus increasing the dynamic range of pixel values to gray to achieve the effect of enhancing the contrast of the entire image. After homogenization the probability of the processed image, the gray-scale all equal, increase the contrast of the image, the image contains the most information.

Histogram equalization principle is as follows:

Let the variable $r$ represents the gray value and assume that it is a continuous variable, where $r$ is normalized, ie, the maximum grayscale value of 1 , $0 \leq r \leq 1$.Let $\varphi(r)$ on behalf of the density function. Now to find a transformed relationship $S=T(r)$, after transformation of the new gray value histogram has a flat.

For the gray-scale transformation meaningful, must require:

(1) in the $0 \leq r \leq 1$ interval, $T(r)$ is a monotonically increasing function, and satisfied $0 \leq T(r) \leq 1$;

(2)the inverse transform $r=T^{-1}(S), 0 \leq S \leq 1$, 
also satisfy the like (1) condition.

This means that the transformed gray remain the order of a single change from black to white, and the range of variation is consistent with the original, in order to prevent the whole image lighter or darker.

Consider the pixel position does not affect the gradation transformation distribution, the number of pixels does not change, so there is:

$$
\begin{aligned}
& \int_{j}^{j+\Delta r} \varphi_{r}(r) d r=\int_{S_{j}}^{S_{j}+\Delta s} \varphi_{s}(S) d S \\
& (\Delta \rightarrow 0 \Delta s \rightarrow 0)
\end{aligned}
$$

By the formula (4) can be deduced

$$
\begin{aligned}
& d S / d r=\varphi_{r}(r) / \varphi_{s}(S) \\
& \text { let } S=T(r) \text { and } \varphi(S)=1, \quad 0 \leq S \leq 1 \quad \text { Into }
\end{aligned}
$$

equation (5), can be obtained

$$
d T(r) / d r=\varphi_{r}(r)
$$

Formula (6) is a gradation transformation formula, $T(r)$ is obviously non-negative function increments, to fully meet the requirements of the transformation function.

Setting a gray-scale image into $L$, the image for the total number of pixels $\mathrm{n}, n_{k}$ behalf of the $\mathrm{K}$-th gray level $r_{k}$ that frequency appears, $P_{r}\left(r_{k}\right)=n_{k} / n$

that, $0 \leq r_{k} \leq 1, \quad k=0,1,2, \ldots L-1$ 。

Discrete form of formula (7) is :

$$
S_{k}=T\left(r_{k}\right)=\sum_{j=0}^{k} n_{j} / n
$$

Can be converted gray level values for each pixel based on the demand balance amount of the image histogram.

Figure 2 is a comparison of the original image and histogram thermometer uniform scale of post.

\section{Image segmentation}

As the thermometer image we get is unevenly distributed multi-grayscale graphics, we want to be useful image information extracted from the image thermometer out, we put this process is called image segmentation. Its purpose is to sense the image of the feature region or the need to apply the extracted feature region.
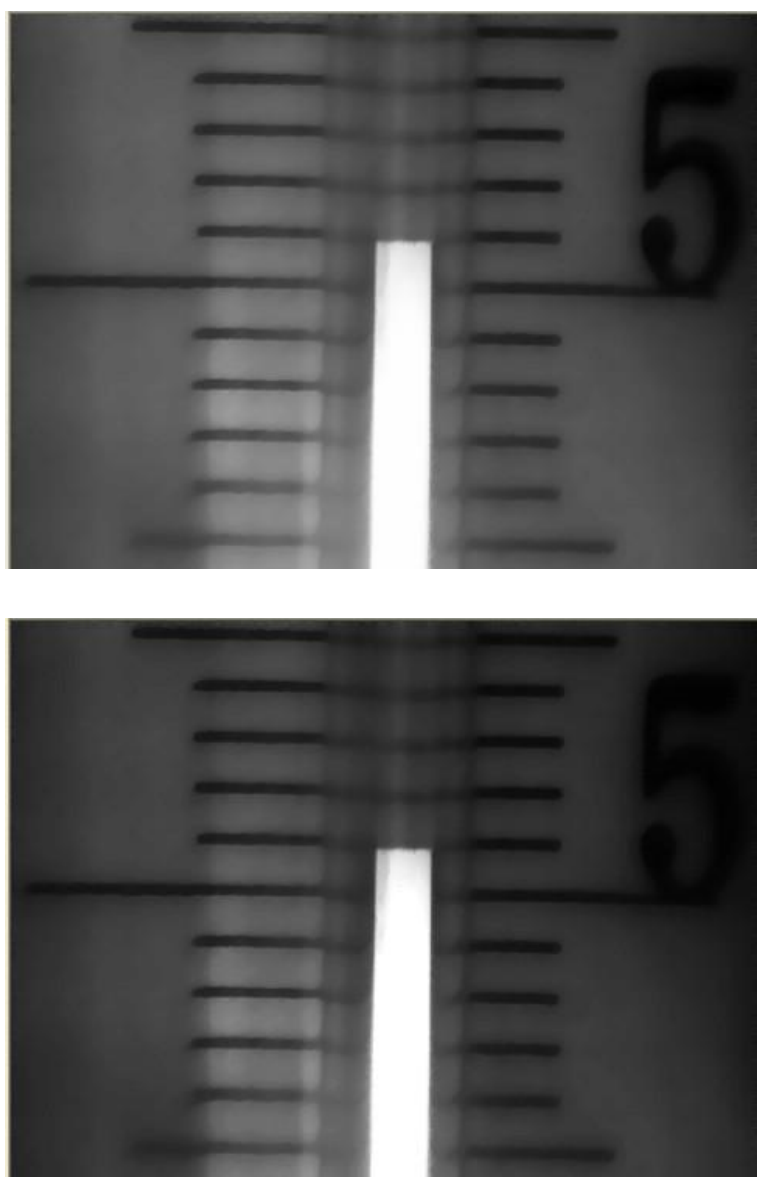

Figure 2. Compares the image before and after histogram equalization

\section{A. Image binarization}

In the process of image segmentation, analyze and understand, the binarized image is the basis of the analysis, it is the image analysis process performed preliminary work. The gray value of each pixel in the $f(x, y)$ image after processing into $B(x, y)$ only two cases 0 or $1, B(x, y)$ is called binary image. Converted by the grayscale image to a binary image is called an image binarization process [3]

Thresholded gray scale image into a binary image converting process, such as the formula (9): 


$$
B(x, y)= \begin{cases}0, & f(x, y)<T \\ 1, & f(x, y) \geq T\end{cases}
$$

\section{B. Comentropy of the Grayscale}

Binary entropy maximization method is Abutaleb in 1989 to develop a one-dimensional to two-dimensional gray gray entropy entropy case, it is the use of two-dimensional gray histogram threshold segmentation method ${ }^{[4]}$.

The principle is: For a $M \times N$ gray image, set its gray level is L, the average gray level in the field this image also is $\mathrm{L}$, neighborhood average gray-scale image is the original image mean filtering the resulting image. Thus the original gray and gray image neighborhood consisting of a gray image tuple $(i, j)$, i represents the original image gradation, $\mathrm{j}$ represents a neighborhood of the gray image, the corresponding frequency is denoted by $C_{i j}$, probability of $(i, j)$ is $P_{i j}=C_{i j} /(M \times N)$; Two-dimensional histogram of the gray target and background in a diagonal position, the rest of the noise and the edges of the image. This article will focus on how to select a threshold on this diagonal, the target and the background area separately.

The amount of information in Shannon's information theory mentioned threshold is applied to the two-dimensional image segmentation threshold ${ }^{[5]}$. Dimensional discrete entropy is:

$$
H=-\sum_{i=1}^{L} \sum_{j=1}^{L} P_{i j} \ln P_{i j}
$$

Single threshold value of the selected two-dimensional $(S, T)$, Target and the background image is divided into two parts, denoted as A and B. The probability of corresponding are: $P_{A}$ and $P_{B}$, respectively $P_{A}=\sum_{i=1}^{S} \sum_{j=1}^{T} P_{i j}, \quad P_{B}=\sum_{i=S+1}^{L} \sum_{j=T+1}^{L} P_{i j}$ 。
$P_{A}$ and $P_{B}$ after the implementation of normalization processing can be expressed as : $\begin{cases}P_{i j}^{\prime}=P_{i j} / P_{A} & i, j \in A \\ P_{i j}^{\prime}=P_{i j} / P_{B} & i, j \in B\end{cases}$

Let the two parts of the two-dimensional discrete entropy, respectively $H(A) 、 H(B)$, the total entropy is $H$, the maximum entropy is $H_{\max }$.

$H(A)=-\sum_{i=1}^{S} \sum_{j=1}^{T} P_{i j}^{\prime} \times \log \left(P_{i j}^{\prime}\right)$

$H(B)=-\sum_{i=S+1}^{L} \sum_{j=T+1}^{L} P_{i j}^{\prime} \times \log \left(P_{i j}^{\prime}\right)$

$H=H(A)+H(B)=-\sum_{i=1}^{L} \sum_{j=1}^{L} P_{i j}^{\prime} \times \ln \left(P_{i j}^{\prime}\right)$

$H_{\text {max }}=\arg \max (H(A)+H(B))$

formula (12)、 (13)、 (14) is substituted into the formula (15) obtained:

$H=\frac{H_{A}}{P_{A}}+\frac{H_{B}}{P_{B}}+\log _{2} P_{A}+\log _{2} P_{B}$

Looking for an appropriate threshold $(S, T)$, So that the maximum entropy $\mathrm{H}$ to obtain a binarized image. Figure 3 is a two-dimensional gray entropy threshold method to get the image binary image. 

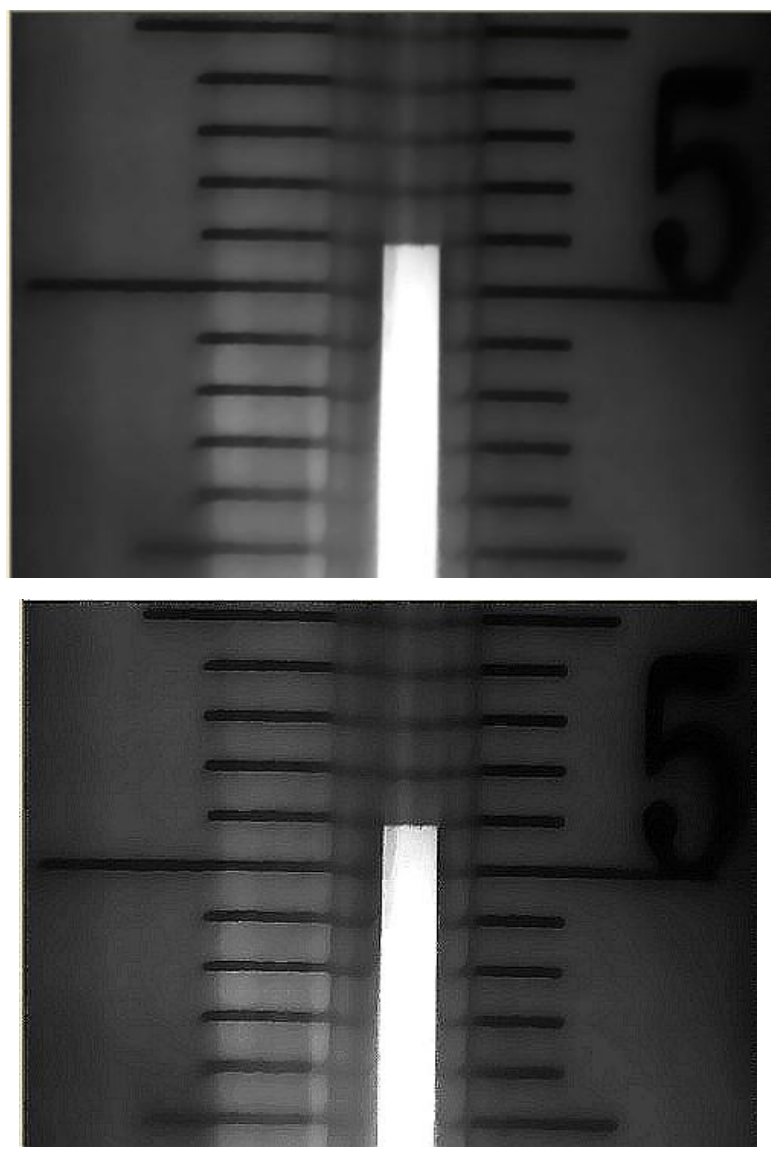

Figure 3. The image is binarized before and after

VI. Conclusions

The main image of thermometer measurement information mentioned herein is included in the plume and the vertical and horizontal distribution of the image scale distribution, they are straight. Theoretically we extract image feature extraction becomes a straight line in the image - horizontal and vertical lines. However, from a severe maldistribution of gray, light and image changing images accurately extract these lines, we must choose the right way of image preprocessing, which is a prerequisite for efficient extraction of feature points.

\section{REFERENCES}

[1] H.A. Rowley, S. Baluja, T. Kanade. Neural Network-Based Face Detection. IEEE Trans. Pattern Anal. Machine Intel. 1998,20(1):23-28

[2] DavisL. Handbook of Genetic \& Algorithm[M]. New
York: van No strand, 1991

[3] Y. Moses, S. Ull man. Limitation of Non-model-based Recognition Schemes. Proc ECCV-92, Sandini G, ed. Springer-Verlag, 1998:820-828

[4] Chen Guo, Zuo Hongfu. 2-D Maximum Entropy Method of Image Segmentation Based on Genetic Algorithm[J].Jouranal of computer-aided Design \& Computer Graphics.2002,14(6):530-534.

[5] A. Grossman, Morlet. Decomposition of Hardy Function Into Square Integrable Wavelets of Constant Shape. 2001,5(15):723-736. 